\title{
Two Planets Straddling the Habitable Zone of the Nearby K Dwarf GI 414A
}

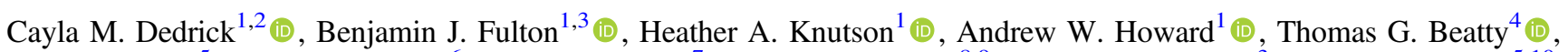
Phillip A. Cargile ${ }^{5}$ (1) B. Scott Gaudi (10), Lea A. Hirsch ${ }^{7}$ (1), Rudolf B. Kuhn ${ }^{8,9}$ (1), Michael B. Lund ${ }^{3}$ (1) David J. James ${ }^{5,10}$ (1),

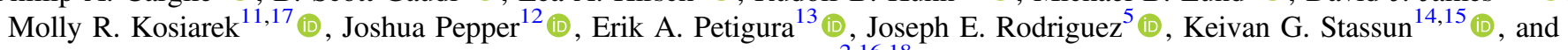
Daniel J. Stevens ${ }^{2,16,18}$ (1)

${ }^{1}$ California Institute of Technology, Pasadena, CA 91125, USA

${ }^{2}$ Department of Astronomy \& Astrophysics, The Pennsylvania State University, 525 Davey Lab, University Park, PA 16802, USA

${ }^{3}$ IPAC-NASA Exoplanet Science Institute, Pasadena, CA 91125, USA

${ }^{4}$ Department of Astronomy and Steward Observatory, University of Arizona, Tucson, AZ 85721, USA

${ }^{5}$ Center for Astrophysics | Harvard \& Smithsonian, 60 Garden Street, Cambridge, MA 02138, USA

${ }^{6}$ Department of Astronomy, The Ohio State University, 140.W. 18th Avenue, Columbus, OH 43210, USA

${ }^{7}$ Kavli Institute for Particle Astrophysics and Cosmology, Stanford University, Stanford, CA 94305, USA

${ }^{8}$ South African Astronomical Observatory, PO Box 9, Observatory, 7935, Cape Town, South Africa

${ }^{9}$ Southern African Large Telescope, PO Box 9, Observatory, 7935, Cape Town, South Africa

${ }^{10}$ Black Hole Initiative at Harvard University, 20 Garden Street, Cambridge, MA 02138, USA

${ }^{11}$ Department of Astronomy and Astrophysics, University of California, Santa Cruz, CA 95064, USA

${ }_{12}^{12}$ Department of Physics, Lehigh University, 16 Memorial Drive East, Bethlehem, PA, 18015, USA

${ }^{13}$ Department of Physics and Astronomy, University of California, Los Angeles, CA 90095, USA

${ }^{14}$ Vanderbilt University, Department of Physics \& Astronomy, 6301 Stevenson Center Lane, Nashville, TN 37235, USA

${ }^{15}$ Fisk University, Department of Physics, 1000 18th Avenue N., Nashville, TN 37208, USA

${ }^{16}$ Center for Exoplanets and Habitable Worlds, The Pennsylvania State University, 525 Davey Lab, University Park, PA 16802, USA

Received 2020 September 11; revised 2020 November 25; accepted 2020 November 27; published 2021 January 28

\begin{abstract}
We present the discovery of two planets orbiting the nearby $(D=11.9 \mathrm{pc}) \mathrm{K} 7$ dwarf Gl 414A. Gl 414A b is a subNeptune mass planet with $M_{b} \sin i_{b}=7.60_{-2.19}^{+2.44} M_{\oplus}$ and a semimajor axis of $0.23 \pm 0.01$ au. Gl 414A c is a subSaturn mass planet with $M_{c} \sin i_{c}=53.83_{-8.58}^{+9.18} M_{\oplus}$ and a semimajor axis of $1.43 \pm 0.06$ au. We jointly analyzed radial velocity data from Keck/HIRES and the Automated Planet Finder at Lick Observatory, as well as photometric data from KELT, to detect the two planets and two additional signals related to the rotationally modulated activity and the long-term magnetic activity cycle of the star. The outer planet in this system may be a potential candidate for future direct-imaging missions.
\end{abstract}

Unified Astronomy Thesaurus concepts: Exoplanets (498); Radial velocity (1332); Gaussian Processes regression (1930); K dwarf stars (876); Exoplanet systems (484)

Supporting material: machine-readable table

\section{Introduction}

High-precision radial velocity (RV) instruments have improved in performance in recent years as the community moves toward the $\sim \mathrm{cm} \mathrm{s}^{-1}$ precision range. When combined with high-cadence observational campaigns, these measurements make it possible to discover new low-mass planets around nearby stars. Statistical methods have also improved greatly, with techniques like Gaussian processes (GPs) to model activity signals (e.g., Dai et al. 2017) making it easier to differentiate between exoplanetary and low-level activityrelated signals (Bastien et al. 2013). The use of a GP ensures that our posteriors for the orbital parameters of the two planets properly account for the RV variations caused by rotationally modulated starspots and other sources of stellar activity.

The Eta-Earth Survey utilized the W. M. Keck Observatory and the HIRES instrument (Vogt et al. 1994) to search 230 of the nearest G-, K-, and M-dwarf stars for low-mass (3-30 $M_{\oplus}$ ) planets (Howard et al. 2009). This survey unveiled the mass distribution of small planets and demonstrated that the occurrence rate of planets is a strong inverse function of their mass (Howard et al. 2010). Our group continues to measure

\footnotetext{
${ }^{17}$ NSF Graduate Research Fellow.

${ }^{18}$ Eberly Research Fellow.
}

RVs for all of the Eta-Earth stars at reduced cadence with both Keck-HIRES and the Automated Planet Finder telescope (APF; Vogt et al. 2014). Some of these stars have been observed for more than two decades, providing sensitivity to planets with orbital periods of many years. At the same time, recent highcadence observing campaigns with the APF have been very important in detecting lower mass planets and disentangling planetary signals from stellar activity (Fulton et al. 2016; Fulton 2017).

The Eta-Earth Survey stars are an ideal test set for utilizing Gaussian processes to model stellar activity in exoplanet detection because of the multidecadal baseline and large number of measurements. We continue to observe stars from the Eta-Earth survey in hopes of finding more small planets with longer orbital periods. Exoplanets with orbital periods greater than 700 days make up less than $10 \%$ of the confirmed planet sample (311 out of 4201). ${ }^{19}$ Due to sensitivity constraints, a majority of these long-period planets are gas giants.

In this paper, we report the discovery of two planets orbiting the nearby K dwarf Gl 414A. This star shows RV variations caused by stellar activity with amplitudes comparable to the planetary signals, which makes it a perfect candidate for

\footnotetext{
${ }^{19}$ https://exoplanetarchive.ipac.caltech.edu/, August 28, 2020.
} 
Table 1

Stellar Properties of Gl 414

\begin{tabular}{|c|c|c|}
\hline Parameter & Value & Source \\
\hline \multicolumn{3}{|l|}{ Gl 414A } \\
\hline \multirow[t]{2}{*}{ Alt. names } & HD 97101 & Cannon \& Pickering (1993) \\
\hline & HIP 54646 & van Leeuwen (2007) \\
\hline RA & 111105.17 & Gaia18 \\
\hline Dec & +302645.66 & Gaia18 \\
\hline Spectral type & $\mathrm{K} 7 \mathrm{~V}$ & Gray et al. (2003) \\
\hline$B-V(\mathrm{mag})$ & 1.255 & Boro Saikia et al. (2018) \\
\hline$m_{V}(\mathrm{mag})$ & $8.864 \pm 0.12$ & Zacharias et al. (2012) \\
\hline$m_{G}(\mathrm{mag})$ & $7.7281 \pm 0.0007$ & Gaia18 \\
\hline$m_{J}(\mathrm{mag})$ & $5.764 \pm 0.033$ & Cutri et al. (2003) \\
\hline$m_{H}(\mathrm{mag})$ & $5.130 \pm 0.033$ & Cutri et al. (2003) \\
\hline$m_{K}(\mathrm{mag})$ & $4.979 \pm 0.033$ & Cutri et al. (2003) \\
\hline Distance (pc) & $11.893 \pm 0.007$ & Gaia18 \\
\hline$T_{\text {eff }}(\mathrm{K})$ & $4120 \pm 109$ & This work (2.1) \\
\hline $\log (g)$ & $4.65 \pm 0.04$ & This work (2.2) \\
\hline$[\mathrm{Fe} / \mathrm{H}]$ & $0.24 \pm 0.10$ & This work (2.1) \\
\hline$[\alpha / \mathrm{Fe}]$ & $0.58_{-0.08}^{+0.02}$ & This work (2.2) \\
\hline$R_{\star}\left(R_{\odot}\right)$ & $0.680 \pm 0.14$ & This work (2.1) \\
\hline$L_{\star}\left(L_{\odot}\right)$ & $0.119 \pm 0.013$ & This work (2.1) \\
\hline$M_{\star}\left(M_{\odot}\right)$ & $0.650 \pm 0.08$ & This work (2.1) \\
\hline Age (Gyr) & $12.4 \pm 5.2$ & This work (2.1) \\
\hline$A_{v}(\mathrm{mag})$ & $0.02_{-0.01}^{+0.04}$ & This work (2.2) \\
\hline \multicolumn{3}{|l|}{ Gl 414B } \\
\hline RA & 111102.54 & Gaia18 \\
\hline Dec & +302641.32 & Gaia18 \\
\hline Spectral type & $\mathrm{M} 2 \mathrm{~V}$ & Stephenson (1986) \\
\hline \multirow[t]{2}{*}{$B-V(\mathrm{mag})$} & $2.41 \pm 0.34$ & Høg et al. (2000); \\
\hline & & Zacharias et al. (2012) \\
\hline$m_{V}(\mathrm{mag})$ & $9.983 \pm 0.01$ & Zacharias et al. (2012) \\
\hline$m_{G}(\mathrm{mag})$ & $9.0471 \pm 0.0011$ & Gaia18 \\
\hline$m_{J}(\mathrm{mag})$ & $6.592 \pm 0.019$ & Cutri et al. (2003) \\
\hline$m_{H}(\mathrm{mag})$ & $5.975 \pm 0.018$ & Cutri et al. (2003) \\
\hline$m_{K}(\mathrm{mag})$ & $5.734 \pm 0.020$ & Cutri et al. (2003) \\
\hline Distance (pc) & $11.877 \pm 0.008$ & Gaia18 \\
\hline$T_{\text {eff }}(\mathrm{K})$ & $3663 \pm 70$ & This work (2.1) \\
\hline$[\mathrm{Fe} / \mathrm{H}]$ & $0.08 \pm 0.09$ & This work (2.1) \\
\hline$R_{\star}\left(R_{\odot}\right)$ & $0.548 \pm 0.017$ & This work (2.1) \\
\hline$L_{\star}\left(L_{\odot}\right)$ & $0.048 \pm 0.005$ & This work (2.1) \\
\hline$M_{\star}\left(M_{\odot}\right)$ & $0.542 \pm 0.022$ & This work(2.1) \\
\hline Age (Gyr) & $11.2 \pm 5.9$ & This work (2.1) \\
\hline
\end{tabular}

(This table is available in its entirety in machine-readable form.)

combining RV and photometry data in order to differentiate between the types of signals. In Section 2, we provide an updated analysis of the properties of the host star. We describe our Doppler measurements from Keck/HIRES and APF/Levy in Section 3, and describe our photometry measurements from KELT in Section 4. In Section 5 we describe our joint analysis of the RV data and photometric data. This includes a Keplerian analysis of significant periodic signals in the RVs and a characterization using GPs to model stellar activity. Section 6 discusses the orbital dynamics, transit, and direct-imaging prospects. We summarize and conclude in Section 7.

\section{Stellar Properties}

Gl 414A, also known as HD 97101A and HIP 54646A, is a bright nearby K7V dwarf star (Gray et al. 2003) at a distance of $11.89 \pm 0.07 \mathrm{pc}$ (Gaia Collaboration et al. 2018, Gaia18 hereafter). Gl 414A has an M2V dwarf companion (Stephenson 1986) with a $V$-band magnitude of 9.98 (Zacharias et al. 2012).
The projected on-sky separation between Gl 414A and Gl 414B is 34.34" (Mason et al. 2001), corresponding to a projected physical separation of $408 \mathrm{au}$. The stars are separated widely enough that spectral contamination from $\mathrm{Gl} 414 \mathrm{~B}$ is not a concern.

\subsection{SpecMatch-Emp and Isoclassify}

We inferred stellar properties using the publicly available software package SpecMatch-Emp (Yee et al. 2017) to quantitatively compare our iodine-free template spectrum of $\mathrm{Gl}$ 414A to a suite of library HIRES spectra for stars with wellmeasured parameters. The SpecMatch-Emp analysis returned $T_{\text {eff }}=4120 \pm 70 \mathrm{~K},[\mathrm{Fe} / \mathrm{H}]=0.24 \pm 0.09$, and $R_{\star}=0.680 \pm$ $0.10 R_{\odot}$.

Since SpecMatch-Emp does not return a stellar mass, we used the isoclassify package (Berger et al. 2020; Huber et al. 2017) to place the star onto MESA Isochrones \& Stellar Tracks (MIST; Choi et al. 2016; Dotter 2016) isochrone tracks. We followed the methodology of Fulton \& Petigura (2018) to derive physical stellar parameters from the spectroscopic parameters. We ran isoclassify in both direct mode and grid mode. In direct mode isoclassify relies on a single apparent magnitude $\left(m_{K}\right.$ in this case), the extinction in that band, the distance modulus, and a bolometric correction obtained by interpolating the MIST/C3K model grid (C. Conroy et al. 2021, in preparation). In grid mode, the star is compared to the isochrones using observational constraints. We informed the isoclassify fit by placing priors on $T_{\text {eff }}$ and $[\mathrm{Fe} / \mathrm{H}]$ from our spectroscopic analysis, the Gaia parallax $(\pi=84.08 \pm 0.471$ mas; Gaia18), and the $K$-band apparent magnitude $\left(m_{K}=4.979 \pm 0.018 \mathrm{mag}\right.$; Cutri et al. 2003). Isoclassify then calculated the posterior probability density for $R_{\star}$ using the Stefan-Boltzmann law. In direct mode, we found that $L_{\star}=0.119 \pm 0.005 L_{\odot}$ and $R_{\star}=0.679 \pm 0.027 R_{\odot}$. Running isoclassify in grid mode also allowed us to extract $M_{\star}=0.650 \pm 0.028 M_{\odot}$ and age $=11.2 \pm 5.9$ Gyr.

We carried out the same analysis to determine stellar properties of the companion, Gl 414B. SpecMatch-Emp returned $T_{\text {eff }}=3663 \pm 70 \mathrm{~K},[\mathrm{Fe} / \mathrm{H}]=0.08 \pm 0.09$, and $R_{\star}=0.515 \pm 0.100 R_{\odot}$. Because Gl 414B is sufficiently cool, isoclassify is able to determine masses in the direct mode using empirical relations from Mann et al. (2019). We found that $R_{\star}=0.548 \pm 0.017 R_{\odot}, M_{\star}=0.542 \pm 0.022 M_{\odot}$, and $L_{\star}=0.048 \pm 0.005 L_{\odot}$. In grid mode, we derive the age as $12.4 \pm 5.2 \mathrm{Gyr}$.

If the two stars formed in the same birth cloud, we would expect the value of $[\mathrm{Fe} / \mathrm{H}]$ and the ages of the two stars to be the same, and indeed the values we derive from SpecMatchEmp and isoclassify analysis are consistent to $1 \sigma$.

We adopt the values of $T_{\text {eff }}$ and $[\mathrm{Fe} / \mathrm{H}]$ from SpecMatchEmp, and the values of $R_{\star}, M_{\star}, L_{\star}$, and age from isoclassify. See Table 1 for the final adopted stellar parameters for both stellar components in the system.

\subsection{MINESweeper}

We also determine stellar parameters using MINESweeper, a tool to model stellar photometry using isochrone priors. Full details and validation of this technique can be found in Cargile et al. (2020), but briefly, the program can fit broadband photometric stellar spectral energy distributions (SEDs) with 
models drawn from the MIST (Choi et al. 2016) stellar isochrones. The SED models (and corresponding predicted photometry) are computed from grids of ATLAS12 model atmospheres (Kurucz 1970) and the spectrum synthesis code SYNTHE (Kurucz 1993). Both atmospheres and SEDs are computed in 1D assuming plane-parallel geometry and LTE. We adopt the solar abundances from Asplund et al. (2009), which is also the abundance scale used in the MIST isochrones. Atomic and molecular line lists are adopted from the latest compilation of R. Kurucz (private communication), and have been astrophysically calibrated against ultra high resolution ( $\mathrm{R}>200 \mathrm{k}$ ) spectra of the Sun and Arcturus using the same model assumptions as adopted herein (Cargile et al. 2021, in preparation). The fit is performed using the nested sampling code dynesty (Speagle 2019).

We fit Gl 414A with MINESweeper using the available photometry from Gaia DR2 (G/BP/RP), the Two Micron AllSky Survey (2MASS) $\left(\mathrm{J} / \mathrm{H} / \mathrm{K}_{s}\right)$, and the Wide-field Infrared Survey Explorer (WISE) (W1/W2/W3). For the Gaia photometry, we assume the filter curves and zero-points defined by Maíz Apellániz \& Weiler (2018) and photometric corrections published on the mission website. ${ }^{20}$

$\mathrm{We}$ find $T_{\text {eff }}=4239_{-83}^{+84}, \quad[\mathrm{Fe} / \mathrm{H}]=+0.25_{-0.05}^{+0.04}, \quad R_{\star}=$ $0.658_{-0.088}^{+0.087}, M_{\star}=0.703_{-0.080}^{+0.079}$, and $L_{\star}=0.126 \pm 0.012$. These results from are consistent with those we get from SpecMatch-Emp and isoclassify. Our modeling suggests that Gl 414A has an enhanced alpha-element abundance $([\alpha / \mathrm{Fe}] \sim+0.6)$.

The MINESweeper analysis gives an age of $2.03_{-0.57}^{+3.77} \mathrm{Gyr}$, which is inconsistent with the isoclassify analysis. However, we note that the age of low-mass stars is very difficult to determine due to their long lifetimes and slow evolution on the main sequence. A more detailed analysis is needed to establish the age of this system but is beyond the scope of this study.

Due to the high signal-to-noise ratio $(\mathrm{S} / \mathrm{N})$ in the spectrum used to perform the spectroscopic analysis $(>350$ per resolution element) the formal statistical uncertainties are extremely small. We have seen from ensemble analysis of spectroscopic extraction codes and comparisons with other techniques that the statistical uncertainties from these codes are dwarfed by the systematic uncertainties in the stellar atmosphere and isochrone models in this high $\mathrm{S} / \mathrm{N}$ regime (e.g., Johnson et al. 2017; Petigura et al. 2017; Pepper et al. 2019).

For this reason, we follow the methodology of Petigura et al. (2017) and Johnson et al. (2017) by adding the following terms in quadrature with our measured Specmatch-Emp+isoclassify uncertainties: $100 \mathrm{~K}$ for $T_{\text {eff }}, 0.1 \mathrm{dex}$ for $\log (g)$, and fractional uncertainties of $2 \%$ for stellar mass, radius, and luminosity. These additional uncertainty terms were found by comparing the parameters estimated by Specmatch-Emp and isoclassify to those from other sources for a large sample of benchmark stars (Yee et al. 2017). In addition, we attempt to incorporate the uncertainty due to our choice of modeling technique by adding the errors from MINESweeper in quadrature with those from SpecMatch-Emp and isoclassify. Our final adopted stellar parameters and associated uncertainties are listed in Table 1.

Cifuentes et al. (2020) derived stellar parameters for Gl 414A and found that $T_{\text {eff }}=4100 \mathrm{~K}, R_{\star}=0.7016 \pm 0.0177 R_{\odot}$,

$\overline{{ }^{20} \text { https://www.cosmos.esa.int/web/gaia/dr2-known-issues }}$
$L_{\star}=1.2530 \pm 0.0016 L_{\odot}$, and $M_{\star}=0.7136 \pm 0.0234 M_{\odot}$. All of these values are in agreement with ours, to within $1 \sigma$. The metallicity measurements in the literature are inconsistent with ours and with one another. Some studies have found that Gl $414 \mathrm{~A}$ is very metal-rich (i.e., Hinkel et al. 2017, [[Fe/ $\mathrm{H}]=0.67$; Luck 2017, $[[\mathrm{Fe} / \mathrm{H}]=0.65])$. However, Houdebine et al. (2016) found that $[\mathrm{Fe} / \mathrm{H}]=-0.20$, using principalcomponent-analysis-based inversion of HARPS spectra. Neves et al. (2013) measured the metallicity photometrically and found that $[\mathrm{Fe} / \mathrm{H}]=0.08$ using photometric calibrations from Johnson $\&$ Apps (2009) or $[\mathrm{Fe} / \mathrm{H}]=-0.11$ using photometric calibrations from Neves et al. (2012). A follow-up study of Gl 414A to measure the detailed chemical abundances is warranted.

Our derived stellar parameters for Gl 414B are consistent with previous measurements from Schweitzer et al. (2019), who found that $T_{\text {eff }}=3753 \pm 51 \mathrm{~K},[\mathrm{Fe} / \mathrm{H}]=0.13 \pm 0.16$, $R_{\star}=0.533 \pm 0.015 R_{\odot}$, and $L_{\star}=0.05079 \pm 0.00057 L_{\odot}$. They also determined the mass of the star using four different methods and found values of $M_{\star}$ ranging from $0.531 M_{\odot}$ to $0.571 M_{\odot}$.

\subsection{Activity Indices}

As indicated earlier, Gl 414A is a relatively active K star. We find that the median metrics of stellar activity as measured by the $\mathrm{Ca}$ II $\mathrm{H} \& \mathrm{~K}$ lines in our Keck/HIRES spectra are $S_{\mathrm{HK}}=0.98 \pm 0.11$ and $\log R_{\mathrm{HK}}^{\prime}=-4.72 \pm 0.05$ (Isaacson \& Fischer 2010). These values suggest a marginally consistent, but perhaps slightly lower, activity level than the values reported in Boro Saikia et al. (2018), who reported $S_{\mathrm{HK}}=1.14$ and $\log R_{\mathrm{HK}}^{\prime}=-4.50$. The discrepancy between $\log R_{\mathrm{HK}}^{\prime}$ from Boro Saikia et al. (2018) and our value is due to differences in the methodology used to convert between $S_{\mathrm{HK}}$ and $\log R_{\mathrm{HK}}^{\prime}$. If we convert their $S_{\mathrm{HK}}$ value to $\log R_{\mathrm{HK}}^{\prime}$ using the methodology of Isaacson \& Fischer (2010) we find a value of $\log R_{\mathrm{HK}}^{\prime}=-4.65$.

\section{Radial Velocity Measurements}

\subsection{Keck/HIRES}

We collected 130 RV measurements between 1997 January 14 and 2020 July 11 using the HIRES instrument on the Keck I telescope (Vogt et al. 1994). This star was observed using the 0 " $86 \times 3$ ". 5 and 0 ". $86 \times 14^{\prime \prime}$ slits for a spectral resolution of $R \approx 65,000$ near $5500 \AA$. We extracted $\mathrm{RV}$ measurements from each spectrum following the technique described in Marcy \& Butler (1992). We observed Gl 414A with a cell of gaseous iodine in the light path just behind the entrance slit. The iodine cell imprints a dense forest of molecular absorption lines onto the stellar spectrum which are used as a simultaneous wavelength and point-spread function (PSF) fiducial. We forward modeled each observation in 718 small chunks of spectral width $\approx 2 \AA$ as

$$
I_{\mathrm{obs}}(\lambda)=k\left[T_{I_{2}}(\lambda) \cdot I_{S}(\lambda+\Delta \lambda)\right] \circledast \mathrm{PSF},
$$

where $T_{I_{2}}$ is the transmission of the iodine cell as measured in a lab and $I_{S}(\lambda+\Delta \lambda)$ is the intrinsic stellar spectrum (perturbed by an RV that produces a wavelength shift of $\Delta \lambda$ ) derived by deconvolving an observed iodine-free stellar spectrum with the instrumental PSF. The product of $T_{I_{2}}$ and $I_{S}$ is then convolved with a model of the PSF which is described by a sum of Gaussians and scaled by an arbitrary normalization factor $k$. We 
Table 2

Gl 414A Radial Velocities

\begin{tabular}{lcccc}
\hline \hline $\begin{array}{c}\mathrm{BJD}_{\mathrm{TDB}} \\
(-2440000)\end{array}$ & $\begin{array}{c}\mathrm{RV} \\
\left(\mathrm{m} \mathrm{s}^{-1}\right)\end{array}$ & $\begin{array}{c}\text { Uncertainty } \\
\left(\mathrm{m} \mathrm{s}^{-1}\right)\end{array}$ & Instrument $^{\mathrm{a}}$ & $S_{\mathrm{HK}}$ \\
\hline 10463.01 & -2.54 & 1.16 & $\mathrm{k}$ & $\ldots$ \\
10546.93 & -21.46 & 1.21 & $\mathrm{k}$ & $\ldots$ \\
13370.08 & 2.23 & 0.90 & $\mathrm{j}$ & 1.04 \\
13425.07 & -4.62 & 0.93 & $\mathrm{j}$ & 1.01 \\
16589.03 & -6.33 & 1.90 & $\mathrm{a}$ & 0.89 \\
16589.04 & -5.30 & 1.80 & $\mathrm{a}$ & 0.93 \\
\hline
\end{tabular}

Note.

${ }^{\mathrm{a}} \mathrm{k}=$ pre-upgrade Keck/HIRES, $\mathrm{j}=$ post-upgrade Keck/HIRES, $\mathrm{a}=\mathrm{APF}$.

list RVs and $\mathrm{Ca}$ II $\mathrm{H}$ and $\mathrm{K}\left(S_{\mathrm{HK}}\right)$ activity indices from Keck/ HIRES in Table 2.

\subsection{APF/Levy}

We collected $376 \mathrm{RV}$ measurements between 2013 October 23 and 2020 April 16 using the Levy spectrograph on the APF telescope (Vogt et al. 2014; Radovan et al. 2014). We used the $1^{\prime \prime} \times 3^{\prime \prime}$ slit for a spectral resolution of $R \approx 100,000$ and reduced the data and extracted velocities in the same way as for Keck/HIRES. RVs and $\mathrm{Ca}$ II $\mathrm{H}$ and $\mathrm{K} S_{\mathrm{HK}}$ activity indices from APF/Levy are also listed in Table 2.

\subsection{Shane/Hamilton Spectrograph}

This star was previously observed by the Twenty-five Year Lick Planet Search (Fischer et al. 2014), with 13 RV measurements collected between 1992 January 17 and 2009 February 3 using the Hamilton Spectrograph on the Shane telescope at Lick Observatory. However, the scatter of these measurements is significantly higher than that of the other two data sets and we find that they add very little information to our fits. Including the Lick data would add several more free parameters to the fit, one for each of the CCD upgrades performed on the Hamilton Spectrograph. For this reason, we elect not to include these measurements in our final analysis.

\section{Visible Light Photometry}

The Kilodegree Extremely Little Telescope (KELT; Pepper et al. 2007) is a small-aperture, wide-field photometric survey searching for transiting planets. The KELT-North telescope is located at Winer Observatory, Arizona, and uses an Apogee AP16E detector with $4096 \times 40969 \mu \mathrm{m}$ pixels and a Mamiya $64580 \mathrm{~mm}$ lens. The KELT field of view is $26^{\circ} \times 26^{\circ}$, with $23^{\prime \prime}$ per pixel, and uses a nonstandard wide bandpass comparable to a broad $R$-band filter.

KELT observed Gl 414A in KELT field KN07, obtaining 9516 photometric measurements between 2006 December 24 and 2013 June 12. These data were reduced and photometry extracted following the procedures described in Siverd et al. (2012). We used the version of the light curve detrended with the trend filtering algorithm (TFA; Kovács et al. 2005). The KELT light curve for Gl 414A has an rms of 7.3 mmag and a median absolute deviation of $6.4 \mathrm{mmag}$. With the large KELT pixels, the nearby companion Gl 414B is fully blended in the KELT aperture and contributes $\sim 20 \%$ to the total flux.

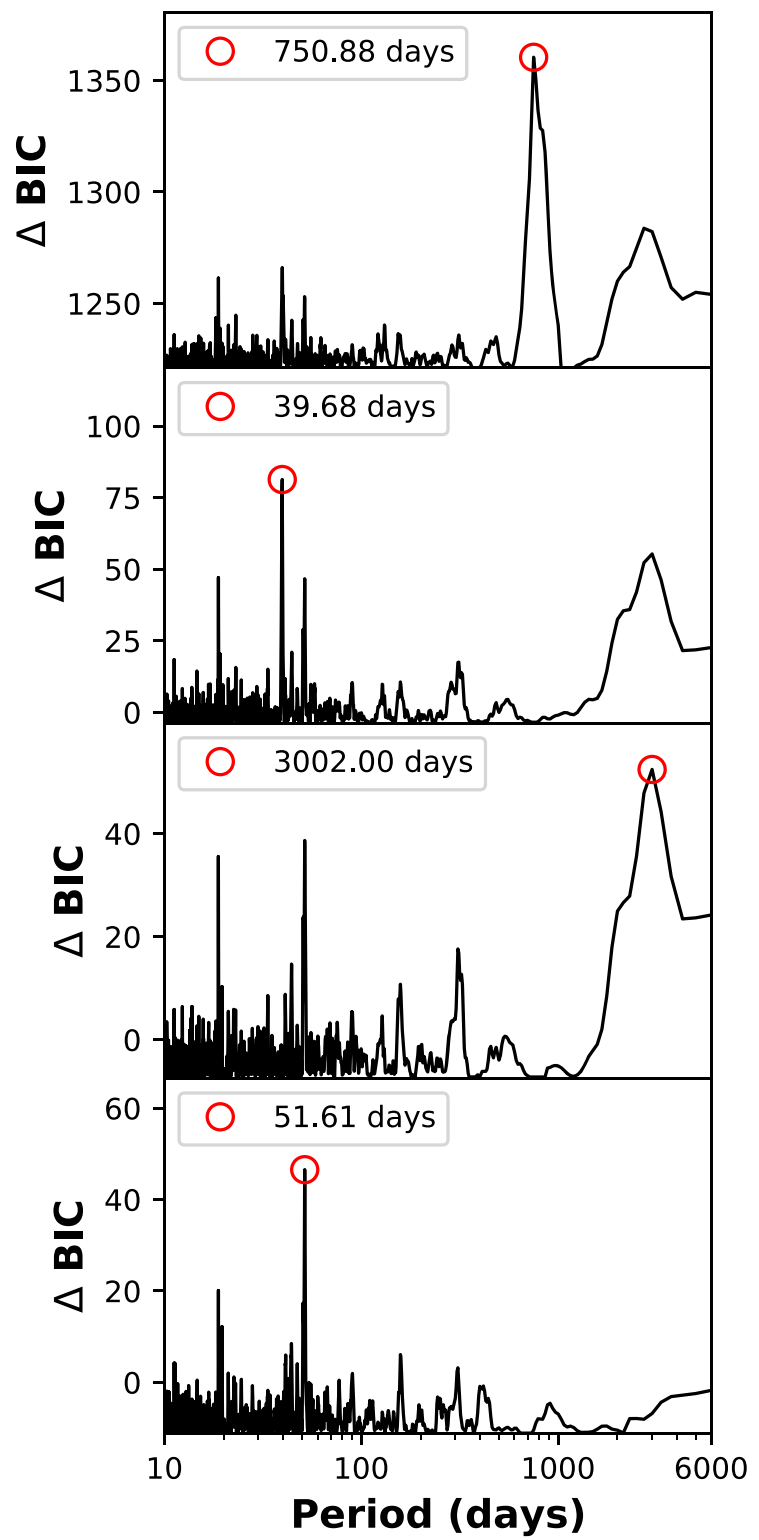

Figure 1. Results of the first four iterations of the multiplanet periodogram search described in Section 5.1. Shown here are the peaks at about 750, 40, 3000 , and 51 days. Each panel has the previous highest signal fixed to look for subsequent signals.

\section{Doppler Analysis}

\subsection{Search for Periodic Signals}

In order to identify significant periodic signals in the RVs, we used a hierarchical approach that compared a Keplerian model with a given number of planets to one with an additional planet to determine if adding a planet improved the quality of the fit at a statistically significant level. The Keplerian model is of the form

$$
\mathcal{V}_{r}=\sum_{k}^{N_{p l}} K_{k}\left[\cos \left(\nu_{k}+\omega_{k}\right)+e_{k} \cos \left(\omega_{k}\right)\right]+\gamma_{i}
$$

where $K$ is the velocity semi-amplitude, $e$ is the eccentricity, $\omega$ is the argument of periapsis, and $\nu$ is the true anomaly given by $\nu=2 \tan ^{-1}\left(\sqrt{\frac{1+e}{1-e}} \tan \frac{E}{2}\right)$, where $E$ is the eccentric anomaly. $\gamma_{i}$ 

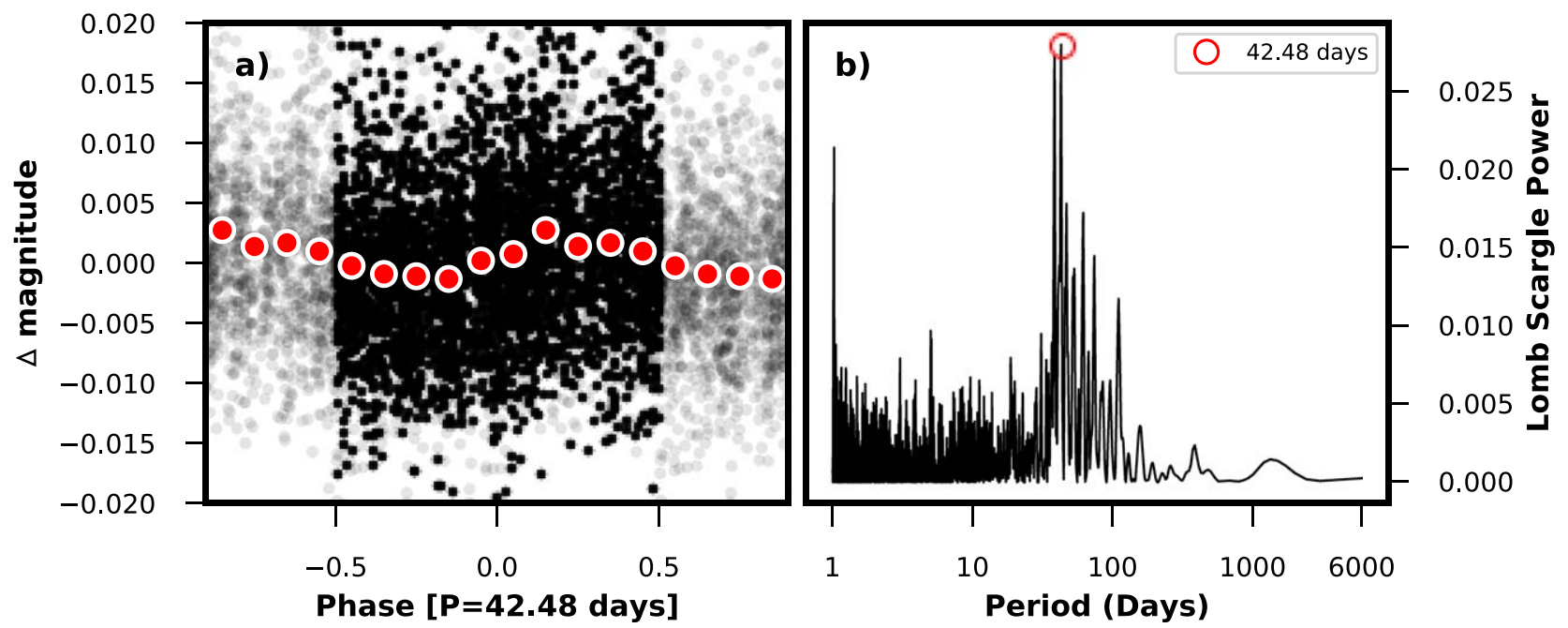

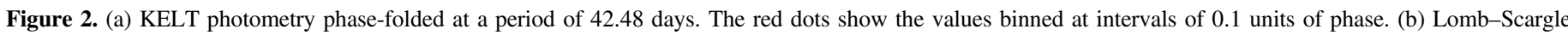
periodogram of the KELT photometry.

is an offset term used to account for the different mean centerof-mass velocities of each instrument $(i)$.

We created a periodogram using the Python package RadVel (Fulton et al. 2018) ${ }^{21}$ version 1.2.13 to fit the RV data with a series of circular Keplerian models with fixed orbital periods. We utilized 3000 search periods evenly spaced in frequency, with values ranging between 10 and 6000 days. At each trial period we kept the period and eccentricity fixed while allowing all other orbital parameters to vary. We fit the model using the maximum a posteriori (MAP) optimization functionality built into RadVel. For fits with $n>2$ planets we kept the orbital parameters of the previously identified planet(s) fixed to the best-fit values from the $n-1$ planet version of the model. After completing our search in period space we represented the power at each trial period as the change in the Bayesian information criterion ( $\triangle \mathrm{BIC}$; Schwarz 1978) with respect to the $n-1$ planet model.

We began our search by comparing a model with one planet to a constant RV and found that the signal with the largest $\triangle \mathrm{BIC}$ was located at 750.88 days. We used this period as our initial guess and fit a new Keplerian orbit where the period and eccentricity were allowed to vary freely. We then incorporated this best-fit model into a new search for a second periodic signal. This revealed another peak at 39.68 days, which we included in a two-planet model that we fit to the data, allowing all parameters for both planets to vary. We repeated this same process for $n$ versus $n-1$ planets until the $\triangle \mathrm{BIC}$ of the highest peak was less than 5 and found significant periodic signals at $750.88,39.68,3002.00,51.61$, and 19.62 days. The first four levels of the periodogram are shown in Figure 1. We note that the 19.62 day peak in the fifth iteration of the periodogram is approximately half of the period of the 39.68 day signal.

\subsection{Stellar Activity}

In order to determine whether or not any of the signals were related to stellar activity, we analyzed 6 years of photometric data from KELT. We used these data to create a Lomb-Scargle (LS; Press \& Rybicki 1989) periodogram (Figure 2), which had

\footnotetext{
21 radvel.readthedocs.io
}

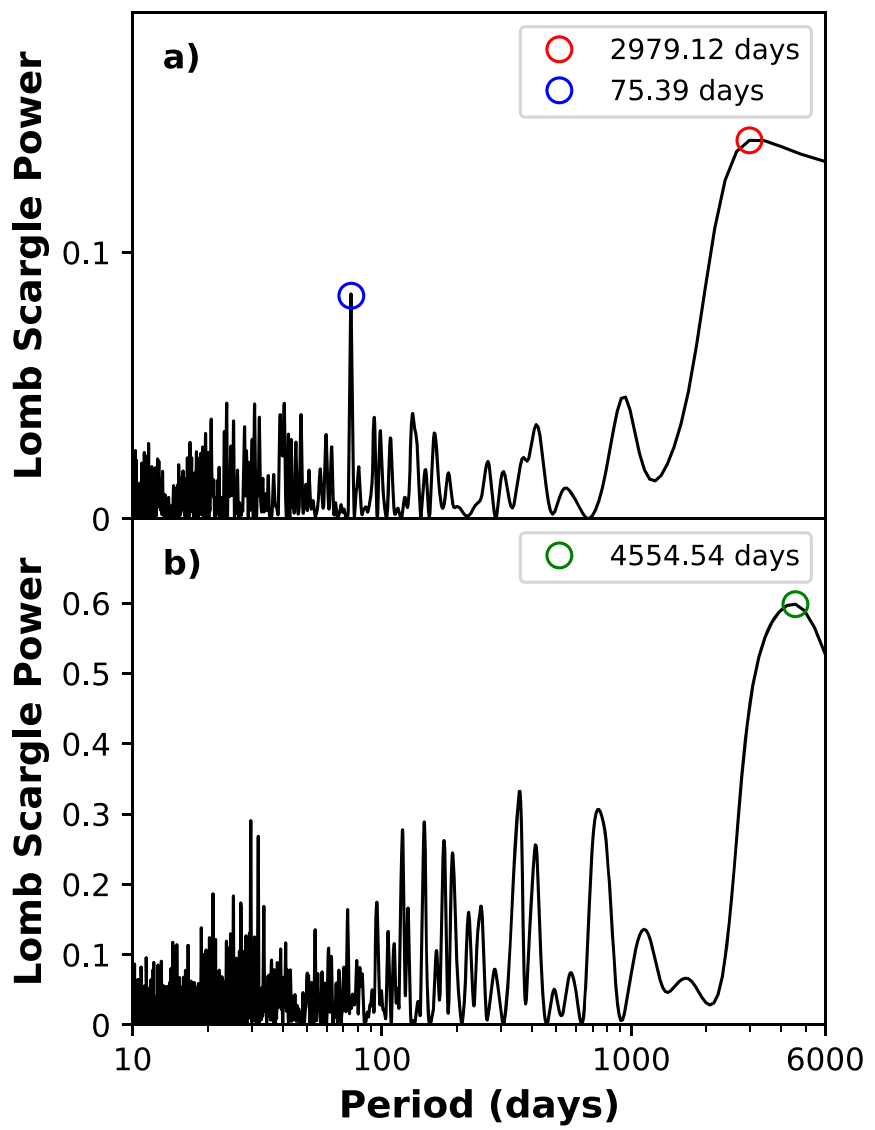

Figure 3. LS periodograms of the $S_{\mathrm{HK}}$ values from (a) APF/Levy and (b) Keck-HIRES. The long-period signals are related to the magnetic activity cycle of the star. The peak at 75 days is approximately two times the 40 day rotation period of the star.

a peak at a period of 42 days. We estimated the season-toseason variance in this period by splitting the data into individual observing seasons and identifying the highest peak for each season. We found that this variance was approximately 4 days.

We also utilized an LS periodogram to search for periodic signals in the $S_{\mathrm{HK}}$ measurements of the star from APF and 

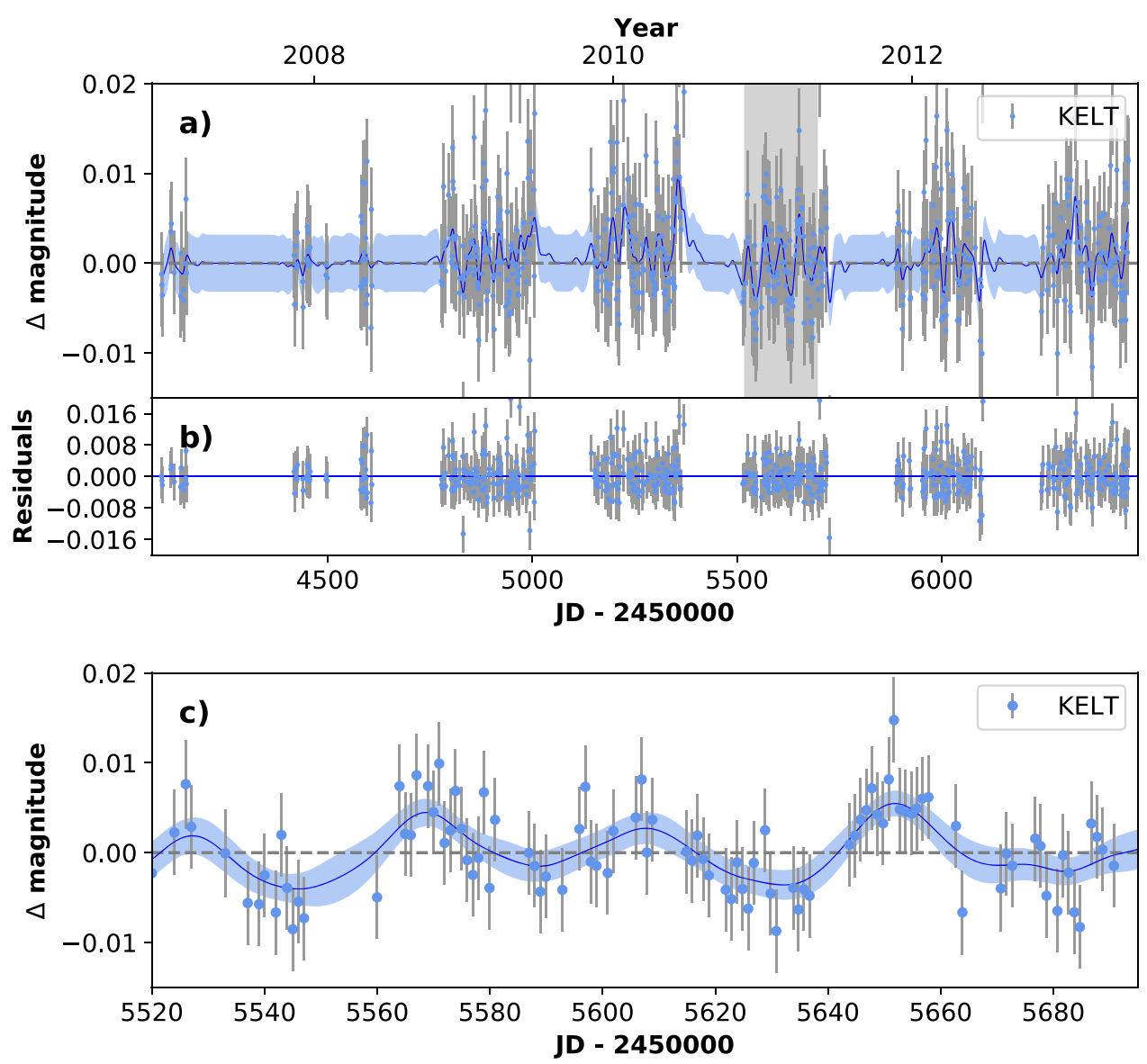

Figure 4. (a) Photometric data from KELT (blue filled circles). The blue line shows the best-fit Gaussian process model, with uncertainties shown as light blue bands. The gray highlighted section is displayed in more detail in panel (c). (b) Fit residuals. (c) A zoomed in view of a single season of photometric data where the quasiperiodic modulations are clearly visible.

Keck-HIRES (Figure 3). We find a peak at 75.4 days in the $S_{\mathrm{HK}}$ measurements of the star from APF. This is approximately twice the period of the 40 day signal in the RV data. When we exclude the last year of measurements, a 40.2 day peak emerges in the LS periodogram. We therefore inferred that the 75.4 day signal appeared due to spot evolution. Because we see similar signals in both the photometry and $S_{\mathrm{HK}}$ data, we concluded that the 39.7 day signal in our RV periodograms was most likely a stellar activity signal related to the stellar rotation period of about 40 days.

In the HIRES and APF periodograms, we found long-period signals at 4554 and 2979 days, respectively, that correspond to the 3000 day signal in the RV data. We concluded that this signal is most likely related to the magnetic activity cycle of the star, which is typically on the order of a decade for FGK stars (Lovis et al. 2011).

\subsection{Orbital Characterization}

We used the affine-invariant Markov Chain Monte Carlo (MCMC) sampler emcee (Foreman-Mackey et al. 2013) to determine the final posterior probability distribution for our two-planet model. This functionality is available as a built-in feature of RadVel. We accounted for the approximately 40 day stellar activity signal using a GP model with a quasiperiodic kernel following a process similar to that described in Kosiarek et al. (2019). We chose not to include the 3002 day signal in this analysis because including it in the model does not significantly change the BIC. The elements of the covariance kernel are of the form

$$
C_{i j}=\eta_{1}^{2} \exp \left[-\frac{\left|t_{i}-t_{j}\right|^{2}}{\eta_{2}^{2}}-\frac{\sin ^{2}\left(\frac{\pi\left|t_{i}-t_{j}\right|}{\eta_{3}}\right)}{2 \eta_{4}^{2}}\right],
$$

where the hyper-parameter $\eta_{1}$ is the amplitude, $\eta_{2}$ is the exponential decay timescale, $\eta_{3}$ is the period, and $\eta_{4}$ is the characteristic length of the periodic component.

The Keplerian and GP likelihoods are given by

$$
\begin{aligned}
& \ln \mathcal{L}_{i}=-\frac{1}{2} \sum_{j}\left[\frac{r_{j}^{2}}{e_{j}^{2}+\sigma_{i}^{2}}-\ln \sqrt{2 \pi\left(e_{j}^{2}+\sigma_{i}^{2}\right)}\right] \\
& \ln \mathcal{L}_{g p}=\frac{1}{2}\left(r^{T} C^{-1} r-\ln (\operatorname{det}(C))-N \ln (2 \pi)\right),
\end{aligned}
$$

respectively, where $r$ is the vector of residuals given by the data $d$ minus the Keplerian model $\mathcal{V}_{r}$ (Equation 2), $e_{j}$ is the error associated with each data point $d_{j}, \sigma_{i}$ is the jitter term for each instrument, $C$ is the covariance matrix, and $N$ is the number of measurements.

The total log-likelihood of all components of the model is then

$$
\ln \mathcal{L}=\sum_{i} \ln \mathcal{L}_{i}+\ln \mathcal{L}_{g p}
$$



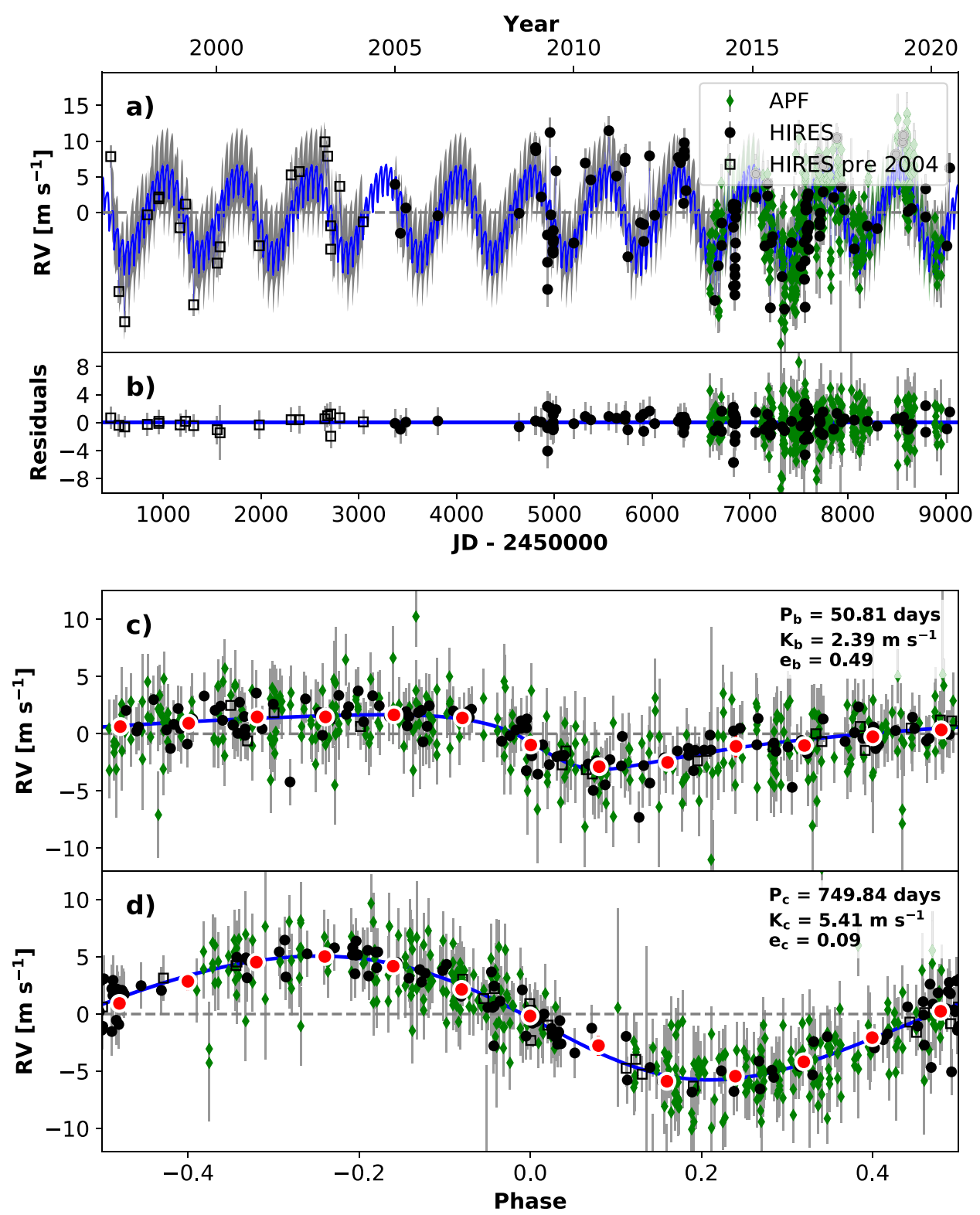

Figure 5. (a) RV time series with the best-fit two-planet model and Gaussian process stellar activity model overplotted in blue. The GP uncertainty is shown as gray bands. (b) Fit residuals. (c) RVs phase-folded to the ephemeris of planet b, with the Keplerian signal of planet c and the GP signal subtracted. Binned data is shown as red dots and the phase-folded model is shown as a blue line. (d) RVs phase-folded to the ephemeris of planet c, with the Keplerian signal of planet $b$ and the GP signal subtracted. Binned data are shown as red dots and the phase-folded model is plotted as a blue line.

We first trained the GP on the photometric data to extract posteriors for the exponential decay length $\left(\eta_{2}\right)$ and GP period $\left(\eta_{3}\right)$. We then repeated this training on the $S_{\mathrm{HK}}$ values from APF using the posteriors from the photometric fit as priors. The priors are applied numerically using a kernel density estimator to approximate the shapes of the posteriors. The resulting posteriors on $\eta_{2}$ and GP period $\eta_{3}$ are then included as priors in the full RV fit. After training on both the photometric and $S_{\mathrm{HK}}$ data sets, we found that $\boldsymbol{\eta}_{2}=31.86_{-11.49}^{+10.67}$ days and $\eta_{3}=42.77_{-5.04}^{+7.33}$ days. We allowed $\eta_{1}$ to vary independently in each fit because the activity-driven amplitudes of the photometry, $S_{\mathrm{HK}}$ values, and RVs are unrelated. We fixed the value of $\eta_{4}$ at 0.5 since allowing it to vary would cause unphysical effects in the fit, as shown in López-Morales et al. (2016). The GP fit to the photometric data in Figure 4 indicates that the 40 day signal is not strictly periodic, as expected for stellar activity. We show the RV data and corresponding bestfit model in Figure 5.

\section{Discussion}

We find compelling evidence for two planets in this system with orbital periods of 750 days and 51 days and minimum masses of $53.83 M_{\oplus}$ and $7.60 M_{\oplus}$, respectively. We attribute the 40 day signal in the RV data to rotationally modulated starspots, and the 3000 day signal to the magnetic activity cycle of the star.

We used the Python code Forecaster, which is based on the probabilistic mass-radius relationship in Chen \& Kipping (2017), to estimate radii corresponding to our minimum mass measurements. We predict that if its orbit is viewed close to 
Table 3

Orbital Parameters

\begin{tabular}{|c|c|c|}
\hline Parameter & Value & Units \\
\hline \multicolumn{3}{|c|}{ Model Parameters } \\
\hline$P_{b}$ & $50.80_{-0.26}^{+0.10}$ & days \\
\hline$T_{\text {conj }, b}$ & $2454203.07_{-6.61}^{+13.06}$ & days \\
\hline$e_{b}$ & $0.45_{-0.22}^{+0.19}$ & \\
\hline$\omega_{b}$ & $1.93_{-0.90}^{+0.63}$ & radians \\
\hline$K_{b}$ & $2.05_{-0.58}^{+0.62}$ & $\mathrm{~m} \mathrm{~s}^{-1}$ \\
\hline$P_{c}$ & $749.83_{-3.63}^{+4.35}$ & days \\
\hline$T_{\text {conj, }, c}$ & $2454210.36_{-26.44}^{+18.31}$ & days \\
\hline$e_{c}$ & $0.105_{-0.703}^{+0.11}$ & \\
\hline$\omega_{c}$ & $1.3_{-3.5}^{+1.3}$ & radians \\
\hline$K_{c}$ & $5.14_{-0.72}^{+0.74}$ & $\mathrm{~m} \mathrm{~s}^{-1}$ \\
\hline$\gamma_{\mathrm{k}}$ & $-10.8 \pm 1.7$ & $\mathrm{~m} \mathrm{~s}^{-1}$ \\
\hline$\gamma_{\mathrm{j}}$ & $-1.62_{-0.77}^{+0.80}$ & $\mathrm{~m} \mathrm{~s}^{-1}$ \\
\hline$\gamma_{\text {apf }}$ & $-0.31 \pm 0.68$ & $\mathrm{~m} \mathrm{~s}^{-1}$ \\
\hline$\sigma_{\mathrm{k}}$ & $0.3 \pm 3.5$ & $\mathrm{~m} \mathrm{~s}^{-1}$ \\
\hline$\sigma_{\mathrm{j}}$ & $1.81_{-0.3}^{+0.37}$ & $\mathrm{~m} \mathrm{~s}^{-1}$ \\
\hline$\sigma_{\text {apf }}$ & $1.26_{-0.3}^{+0.25}$ & $\mathrm{~m} \mathrm{~s}^{-1}$ \\
\hline$\eta_{1, \mathrm{k}}$ & $5.5_{-2.0}^{+1.7}$ & $\mathrm{~m} \mathrm{~s}^{-1}$ \\
\hline$\eta_{1, \mathrm{j}}$ & $4.77_{-0.54}^{+0.61}$ & $\mathrm{~m} \mathrm{~s}^{-1}$ \\
\hline$\eta_{1, \text { apf }}$ & $4.1_{-0.39}^{+0.45}$ & $\mathrm{~m} \mathrm{~s}^{-1}$ \\
\hline$\eta_{2}$ & $40.5_{-11.0}^{+8.7}$ & \\
\hline$\eta_{3}$ & $40.3_{-2.1}^{+2.4}$ & \\
\hline \multicolumn{3}{|c|}{ Derived Parameters } \\
\hline$\sqrt{e_{b}} \sin \omega_{b}$ & $0.50_{-0.38}^{+0.22}$ & \\
\hline$\sqrt{e_{b}} \cos \omega_{b}$ & $-0.27_{-0.29}^{+0.38}$ & \\
\hline$a_{b}$ & $1.400_{-0.060}^{+0.055}$ & $\mathrm{AU}$ \\
\hline$M_{b} \sin i$ & $7.60_{-2.19}^{+2.44}$ & $M_{\oplus}$ \\
\hline$R_{b}$ & $2.63_{-0.85}^{+1.22}$ & $R_{\oplus}$ \\
\hline$T_{\mathrm{eq}, b}$ & $308.6 \pm 33.5$ & $\mathrm{~K}$ \\
\hline$\sqrt{e_{c}} \sin \omega_{c}$ & $0.11_{-0.25}^{+0.22}$ & \\
\hline$\sqrt{e_{c}} \cos \omega_{c}$ & $-0.11_{-0.23}^{+0.26}$ & \\
\hline$a_{c}$ & $0.2324_{-0.0099}^{+0.0092}$ & $\mathrm{AU}$ \\
\hline$M_{c} \sin i$ & $53.83_{-8.58}^{+9.18}$ & $M_{\oplus}$ \\
\hline$R_{c}$ & $8.40_{-2.50}^{+3.60}$ & $R_{\oplus}$ \\
\hline$T_{\mathrm{eq}, c}$ & $124.7 \pm 13.5$ & $\mathrm{~K}$ \\
\hline
\end{tabular}

edge-on, planet b should have a radius of $\boldsymbol{R}_{\boldsymbol{b}}=2.63_{-0.85}^{+1.22} \boldsymbol{R}_{\oplus}$ and planet $\mathrm{c}$ should have a radius of $\boldsymbol{R}_{c}=8.40_{-2.50}^{+3.60} R_{\oplus}$. Assuming that the bond albedo of planet $\mathrm{b}$ is equal to the mean total albedo of super-Earths $\left(A_{t}=0.32\right.$; Demory 2014) and planet c's albedo is equal to that of Saturn $\left(A_{t}=0.343\right.$; Mallama et al. 2017), we calculate predicted equilibrium temperatures of $T_{\mathrm{eq}, b}=308.6 \pm 33.5 \mathrm{~K}$ and $T_{\mathrm{eq}, c}=$ $124.7 \pm 13.5 \mathrm{~K}$ for these two planets. We list the full set of derived properties for each planet in Table 3 .

\subsection{Orbital Dynamics}

We simulated the system using the REBOUND $N$-body integration code (Rein \& Liu 2012; Rein \& Tamayo 2015) to check for stability and investigate the orbital dynamics. We initially assumed that the orbits are coplanar and set the orbital elements to their MAP values. We simulated the system in this configuration for 10 million years with a time step of 0.17 days. The system remained stable in this configuration throughout the simulation. Figure 6 shows the evolution of eccentricity and the argument of periastron $(\omega)$ as a function of time for the first 50,000 years of the simulation. $\omega_{b}$ precesses at a relatively
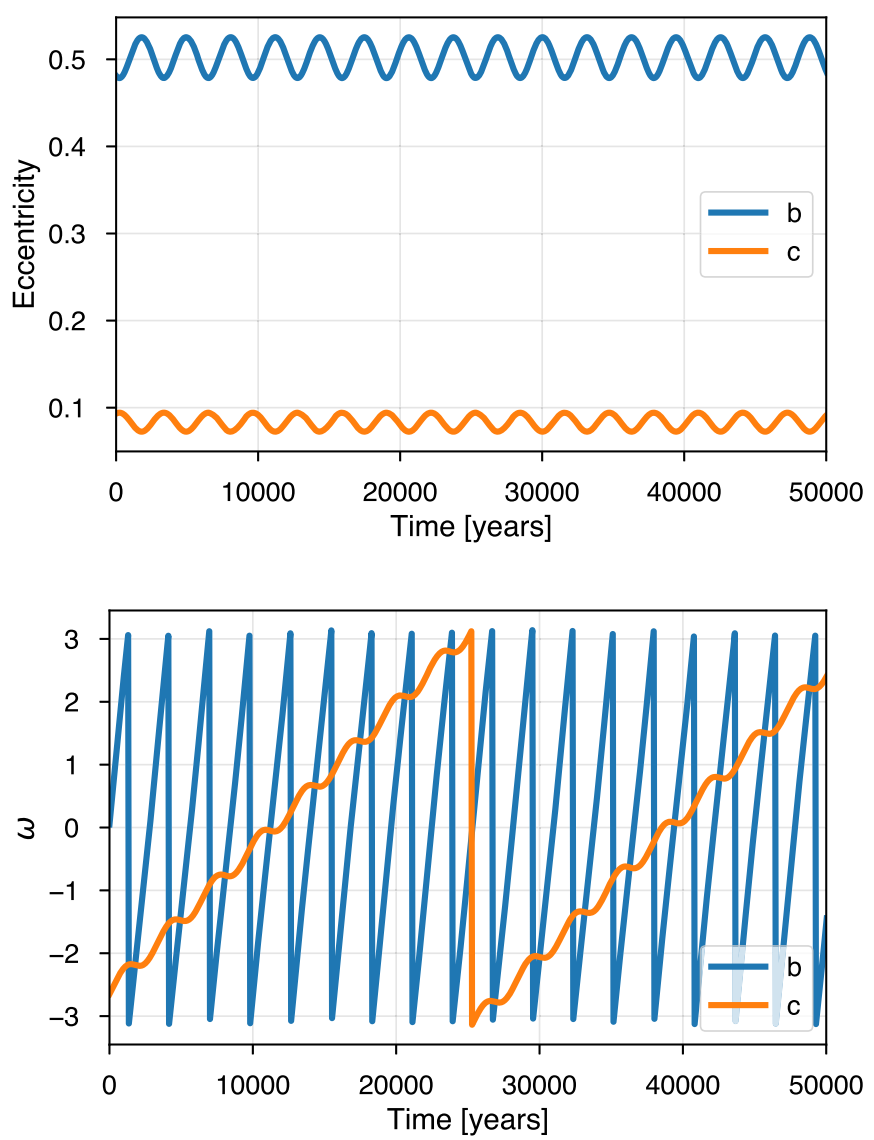

Figure 6. Evolution of orbital parameters from a $N$-body simulation assuming coplanar orbits. Top: eccentricity as a function of time for each planet. Bottom: argument of periastron $(\omega)$ for each planet as a function of time. Each planet's eccentricity oscillates slightly as $\omega_{b}$ precesses with a timescale of $\approx 2.5 \mathrm{kyr}$. $\omega_{c}$ preccesses more slowly, with a timescale of $\approx 25 \mathrm{kyr}$.

rapid rate of 360 degrees every $\sim 2500 \mathrm{yr}$. The eccentricities of both planets trade off with each other on this same timescale. $\omega_{c}$ precesses more slowly, with a period of $\sim 25,000 \mathrm{yr}$, but the rate of precession oscillates at the shorter timescale ( $\sim 2500 \mathrm{yr})$. To test the sensitivity of these precession rates to the initial conditions we also draw 10 sets of orbital parameters from the posterior distributions as starting points for a suite of $N$-body simulations. We find that the precession timescales vary by $\sim 30 \%$, but the general properties of the orbital evolution remain constant.

We measure a significant eccentricity for the inner planet $\left(e_{b}=0.45_{-0.22}^{+0.19}\right)$ while the outer, more massive planet is on a nearly circular orbit. The relatively large eccentricity of the inner planet in this system might plausibly be due to some kind of past dynamical instability (Huang et al. 2017; Carrera et al. 2019). If the two planets have a mutual inclination greater than $\sim 45^{\circ}$, the inner planet could also be due to Kozai-Lidov oscillations where it cycles between states with high inclination and high eccentricity (Kiseleva et al. 1998). However, for planets close to their host stars, general relativistic (GR) precession may also be significant and can dampen the amplitude of the Kozai-Lidov oscillations (e.g., Ford et al. 2000; Fabrycky \& Tremaine 2007). Following the methodology of Yee et al. (2018), we calculate the GR and Kozai timescales for planet $b$. We find that the GR timescale is $\tau_{\mathrm{GR}} \sim 5 \mathrm{Myr}$ and the Kozai timescale is $\tau_{\text {Kozai }} \sim 24 \mathrm{kyr}$. Since 


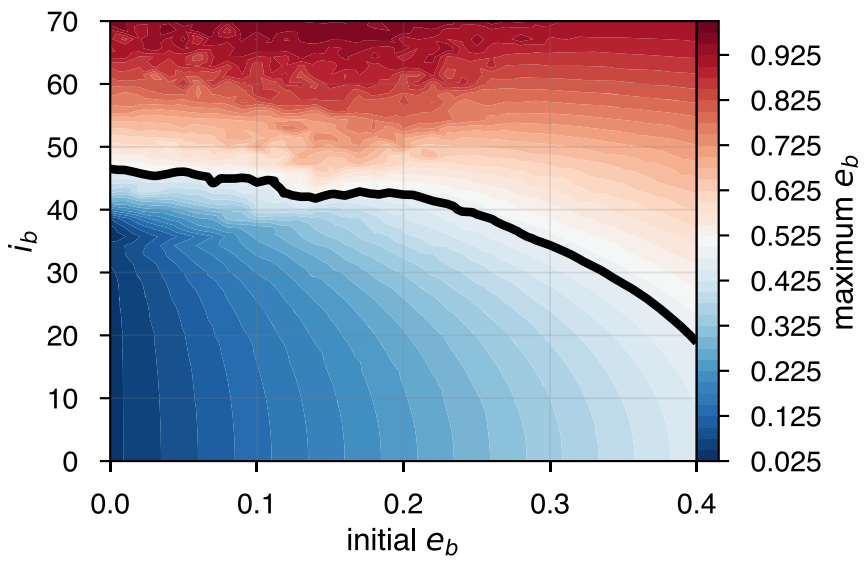

Figure 7. Grid of $N$-body simulations. Each simulation starts with a different initial eccentricity and inclination for the inner planet. The color scale shows the maximum eccentricity reached by the inner planet during each $50 \mathrm{kyr}$ simulation. The thick black lines show the minimum combination of initial eccentricity and mutual inclination needed to explain the current eccentricity of planet b.

$\tau_{\text {Kozai }} \ll \tau_{\mathrm{GR}}$ it is possible that this system is currently undergoing Kozai oscillations.

We explored the impact of mutual inclination on the system architecture by running many REBOUND simulations, each time perturbing the inclination and initial eccentricity of the inner planet keeping the other orbital elements fixed to their MAP values. For each simulation we recorded the maximum eccentricity reached by the inner planet during the 50,000 year simulation (Figure 7). We find that if the orbit of the inner planet was initially circular, the mutual inclination of the system must be $\gtrsim 45$ degrees in order to explain the current eccentricity of planet $b$.

For planets that come close to the star during periastron, tidal circularization is expected to damp out the eccentricity induced by Kozai oscillations or other dynamical interactions (e.g., Fabrycky \& Tremaine 2007). We estimated the tidal circularization timescales for this system using the method outlined in Albrecht et al. (2012). Planets b and c both have extremely long tidal circularization times, at $2.4 \times 10^{11} \mathrm{Gyr}$ and $2.7 \times$ $10^{12} \mathrm{Gyr}$ respectively. We therefore expect that any dynamically induced orbital eccentricity should persist to the present day.

\subsection{Transit Search}

We calculate a priori transit probabilities of $1.3 \%$ and $0.2 \%$ for planets $\mathrm{b}$ and $\mathrm{c}$, respectively. The a posteriori transit probabilities are likely slightly higher (Stevens \& Gaudi 2013), but it is unlikely that either of them transit. If they were to transit, we can use the radii estimates from the previous section to calculate predicted transit depths of $0.1 \%$ and $1.0 \%$ after accounting for $20 \%$ dilution in the KELT photometry due to $\mathrm{Gl}$ 414B. We plot the KELT photometry phase-folded on the ephemeris of each planet in Figure 8. We conclude that there is no evidence to suggest that either planet is transiting. However, given the amplitude of the noise in the KELT photometry and the small predicted transit depth of Gl 414A b, more precise photometry would be needed to rule out the possibility entirely.

\subsection{Direct-imaging Prospects}

At a distance of just $12 \mathrm{pc}$, this relatively cool star is a promising prospect for direct-imaging studies. We find that the planets in this system have projected separations of 20 and 120 mas.
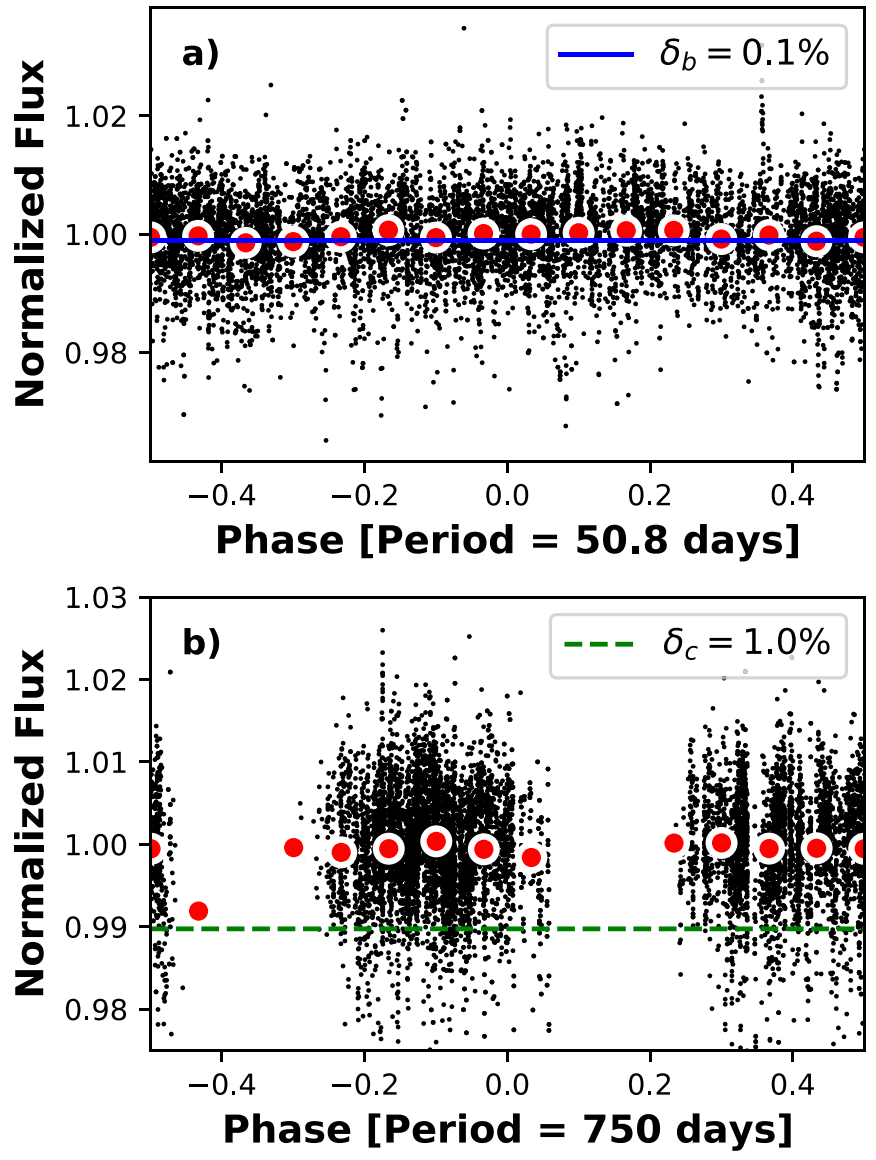

Figure 8. Normalized KELT photometry phase-folded on the ephemeris of (a) Gl 414A b and (b) Gl 414A c. The blue line and green dashed line indicate the predicted transit depths of planets $b$ and $c$ respectively.

We first compare these separations to the inner working angle (IWA) of two ground-based direct-imaging instruments. The Keck Planet Imager and Characterizer (KPIC; Mawet et al.

2018) has an IWA of 40-100 mas, and the SpectroPolarimetric High contrast imager for Exoplanets REsearch (SPHERE; Beuzit et al. 2019) has an IWA of 50-80 mas. Gl $414 \mathrm{~A} \mathrm{c}$ is beyond the IWA of all of these instruments. However, in the $\mathrm{K}$ band, the contrast would be $2.5 \times 10^{-11}$, which rules out ground-based instruments that operate in the near-infrared, like KPIC and SPHERE.

We also compare these angles to the IWA of the James Webb Space Telescope (JWST) coronagraphs. The only mask with an IWA less than the separation of Gl 414Ac is the nonredundant mask (NRM) on the Near Infrared Imager and Slitless Spectrograph (NIRISS). The minimum IWA of the NRM is 89 mas, when observing with the F277W filter. At $2.77 \mu \mathrm{m}$, the contrast of planet $\mathrm{c}$ with respect to its host star is $1.27 \times 10^{-7}$. At this contrast, Gl $414 \mathrm{~A} \mathrm{c}$ is not visible to NIRISS (Doyon et al. 2012).

However, given its contrast in the mid-infrared $\left(10^{-6}\right.$ at $10 \mu \mathrm{m})$ this planet may be a candidate in the future for imaging with a mid-infrared adaptive optics system on a $30 \mathrm{~m}$ class telescope (Chun et al. 2006).

\subsection{Habitability}

Using the model described in Kopparapu et al. (2013), we calculate that the habitable zone around this star should lie between 0.37 and 0.70 au. Planet c falls well outside this zone, 


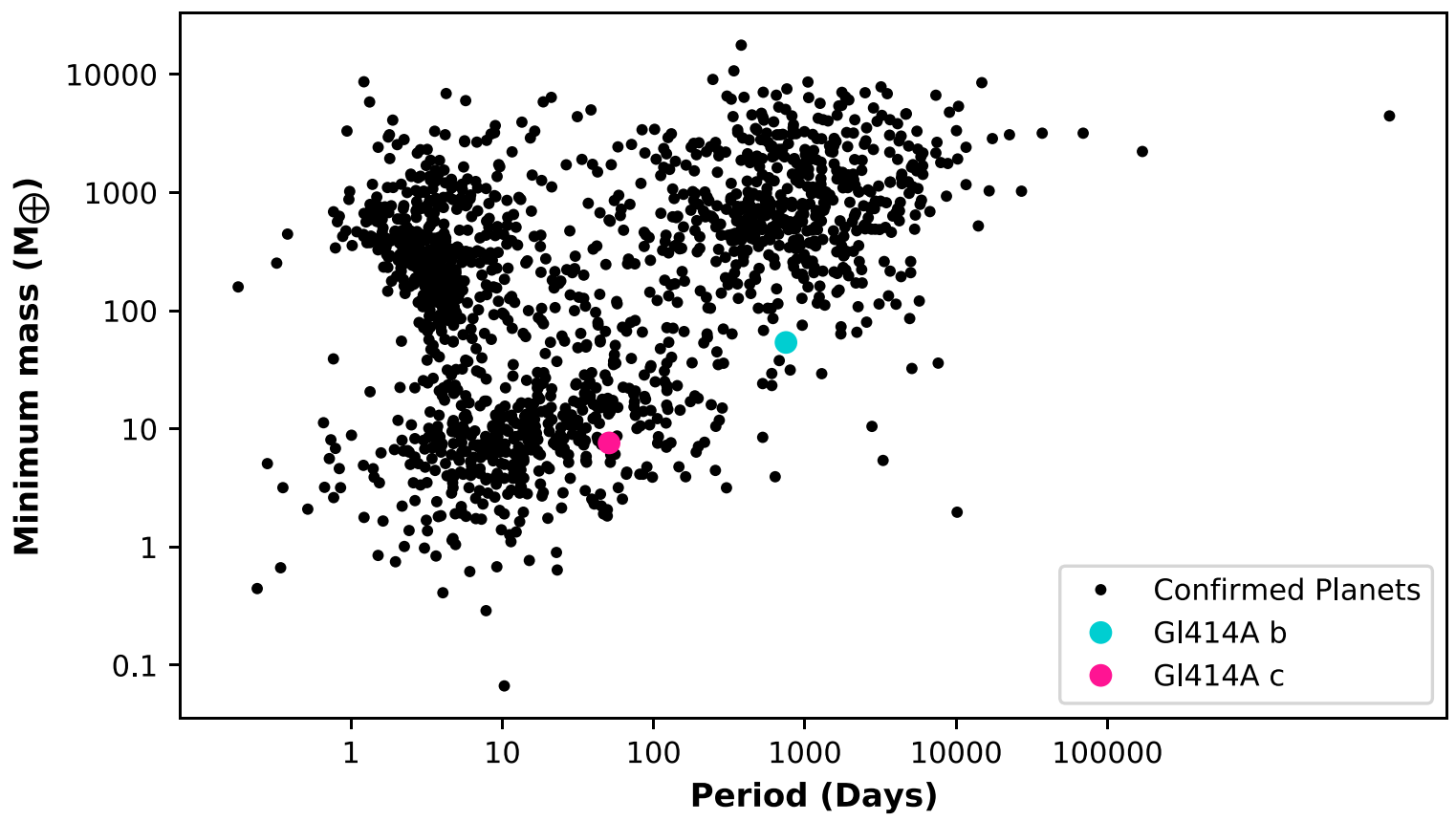

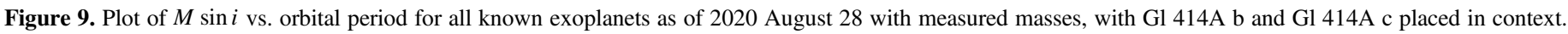
This plot illustrates that these two planets are among the longest period planets that have been detected to date in their respective mass ranges.

but planet $\mathrm{b}$ is just inside this inner edge. If we use the more optimistic model from Zsom et al. (2013), the inner edge of the habitable zone may be as close as 0.21 au. With a semimajor axis of $0.23 \mathrm{au}$ and an eccentricity of 0.45 , Gl $414 \mathrm{~A} \mathrm{~b}$ has a planet distance that varies between 0.13 and 0.34 au and it would fall within this habitable zone range during much of its orbit. However, planet b has a minimum mass of 7.6 $M_{\oplus}$ which likely corresponds to a substantial volatile-rich envelope (Weiss \& Marcy 2014), so it is not a good candidate for habitability.

\section{Summary and Conclusion}

We present the discovery of a sub-Neptune planet and a subSaturn planet orbiting the bright K7 dwarf Gl 414A. The minimum masses of the planets are $\boldsymbol{M}_{\boldsymbol{b}} \sin i_{\boldsymbol{b}}=7.60_{-2.19}^{+2.44} \boldsymbol{M}_{\oplus}$ and $\boldsymbol{M}_{\boldsymbol{c}} \sin i_{\boldsymbol{b}}=53.83_{-8.58}^{+9.18} \boldsymbol{M}_{\oplus}$ and they orbit with semimajor axes $\boldsymbol{a}_{\boldsymbol{b}}=0.23 \pm 0.01$ au and $\boldsymbol{a}_{\boldsymbol{c}}=1.40 \pm 0.01 \mathrm{au}$. Planet $\mathrm{b}$ resides near the inner edge of the star's habitable zone, but its minimum mass is large enough that it likely possesses a substantial volatile-rich envelope. Figure 9 shows the period versus mass of all currently known planets detected using the $\mathrm{RV}$ technique and places $\mathrm{Gl} 414 \mathrm{~A} \mathrm{~b}$ and $\mathrm{Gl} 414 \mathrm{~A} \mathrm{c}$ in context.

In a search of the 4201 confirmed planets listed in the NASA Exoplanet Archive as of 2020 August 28, we found eight other planets in multiplanet systems where one of the planets had an orbital period between 30 and 100 days and an eccentricity greater than 0.4. (HD 163607, Kepler-419, HD 168443, HD 37605, Kepler-432, HD 74156, V1298 Tau, and HD $147018){ }^{22}$ In seven of these systems, there are exactly two planets detected. The high-eccentricity planet is always the inner planet, and the outer planet always has a large separation from the inner planet $(P>400$ days). This suggests that this class of planets may have a common dynamical origin; for example, it is possible that these system architectures all arose

\footnotetext{
${ }^{22}$ https://exoplanetarchive.ipac.caltech.edu/
}

from Kozai-Lidov oscillations. These systems may represent a dynamically active subset of the larger exoplanet population.

We are grateful to the time assignment committees of the California Institute of Technology, the University of California, the University of Hawaii, and NASA for their generous allocations of observing time. Without their long-term commitment to RV monitoring, these planets would likely remain unknown. We gratefully acknowledge the efforts and dedication of the staffs of the W. M. Keck Observatory and Mt. Hamilton. Research at the Lick Observatory is partially supported by a generous gift from Google. We thank Geoff Marcy and Debra Fischer for their many nights of observing that contributed to the Keck data presented in this work, and R. Paul Butler and S. S. Vogt for many years of contributing to the data presented here. C.M.D. acknowledges the Caltech Summer Undergraduate Research Fellowship program along with partial support from the family of Samuel P. and Frances Krown. A.W.H. acknowledges NSF grant AST-1517655. M.R.K. acknowledges support from the NSF Graduate Research Fellowship, grant No. DGE 1339067. D.J.S. was supported as an Eberly Research Fellow by the Eberly College of Science at the Pennsylvania State University.

The authors extend special thanks to those of Hawaiian ancestry on whose sacred mountain of Maunakea we are privileged to be guests. Without their generous hospitality, the Keck observations presented herein would not have been possible.

This work made use of the GNU Parallel package for largescale multiprocessing (Tange 2011). This research has made use of the NASA Exoplanet Archive, which is operated by the California Institute of Technology, under contract with the National Aeronautics and Space Administration under the Exoplanet Exploration Program. This work made extensive use of the scipy (Virtanen et al. 2020), numpy (van der Walt et al. 2011), matplotlib (Hunter 2007), and pandas (McKinney 2010) Python packages. 
Simulations in this paper made use of the REBOUND code which is freely available at http://github.com/hannorein/ rebound.

Facilities: Automated Planet Finder (Levy), Keck:I (HIRES), KELT, Exoplanet Archive.

Software: SpecMatch-Emp (Yee et al. 2017), isoclassify (Berger et al. 2020; Huber et al. 2017), MIST (Dotter 2016; Choi et al. 2016), MINESweeper (Cargile et al. 2020), ATLAS12 (Kurucz 1970), SYNTHE (Kurucz 1993), dynesty (Speagle 2019), RadVel (v1.2.13; Fulton et al. 2018), emcee (Foreman-Mackey et al. 2013), Forecaster (Chen \& Kipping 2017), REBOUND (Rein \& Liu 2012; Rein \& Tamayo 2015), Astropy (Astropy Collaboration et al. 2013, 2018), scipy (Virtanen et al. 2020), numpy (van der Walt et al. 2011), matplotlib (Hunter 2007), pandas (McKinney 2010).

\section{ORCID iDs}

Cayla M. Dedrick ํㅏ https://orcid.org/0000-0001-9408-8848 Benjamin J. Fulton (1) https://orcid.org/0000-0003-3504-5316 Heather A. Knutson (1) https://orcid.org/0000-0002-5375-4725 Andrew W. Howard (1) https://orcid.org/0000-0001-8638-0320 Thomas G. Beatty (ib https://orcid.org/0000-0002-9539-4203 Phillip A. Cargile iㅏ https://orcid.org/0000-0002-1617-8917 B. Scott Gaudi (10) https://orcid.org/0000-0003-0395-9869 Lea A. Hirsch (1) https://orcid.org/0000-0001-8058-7443 Rudolf B. Kuhn (ib https://orcid.org/0000-0002-4236-9020 Michael B. Lund (ㅇ) https://orcid.org/0000-0003-2527-1598 David J. James (1) https://orcid.org/0000-0001-5160-4486 Molly R. Kosiarek (1) https://orcid.org/0000-0002-6115-4359 Joshua Pepper (1) https://orcid.org/0000-0002-3827-8417 Erik A. Petigura (ib https://orcid.org/0000-0003-0967-2893 Joseph E. Rodriguez (i) https://orcid.org/0000-00018812-0565

Keivan G. Stassun (ib https://orcid.org/0000-0002-3481-9052 Daniel J. Stevens (i) https://orcid.org/0000-0002-5951-8328

\section{References}

Albrecht, S., Winn, J. N., Johnson, J. A., et al. 2012, ApJ, 757, 18 Asplund, M., Grevesse, N., Sauval, A. J., \& Scott, P. 2009, ARA\&A, 47, 481 Astropy Collaboration, Price-Whelan, A. M., SipHocz, B. M., et al. 2018, AJ, 156,123

Astropy Collaboration, Robitaille, T. P., Tollerud, E. J., et al. 2013, A\&A, 558, A33

Bastien, F. A., Stassun, K. G., Basri, G., \& Pepper, J. 2013, Natur, 500, 427 Berger, T. A., Huber, D., van Saders, J. L., et al. 2020, AJ, 159, 280 Beuzit, J. L., Vigan, A., Mouillet, D., et al. 2019, A\&A, 631, A155 Boro Saikia, S., Marvin, C. J., Jeffers, S. V., et al. 2018, A\&A, 616, A108 Cannon, A. J., \& Pickering, E. C. 1993, yCat, 3135, 0 Cargile, P. A., Conroy, C., Johnson, B. D., et al. 2020, ApJ, 900, 28 Carrera, D., Raymond, S. N., \& Davies 2019, A\&A, 629, L7 Chen, J., \& Kipping, D. 2017, ApJ, 834, 17

Choi, J., Dotter, A., Conroy, C., et al. 2016, ApJ, 823, 102

Chun, M. R., Elias, J., Ellerbroek, B., et al. 2006, Proc. SPIE, 6272, 62720S Cifuentes, C., Caballero, J. A., Cortés-Contreras, M., et al. 2020, A\&A, 642, A115

Cutri, R. M., Skrutskie, M. F., van Dyk, S., et al. 2003, yCat, 2246, 0

Dai, F., Winn, J. N., Gandolfi, D., et al. 2017, AJ, 154, 226

Demory, B.-O. 2014, ApJL, 789, L20

Dotter, A. 2016, ApJS, 222, 8

Doyon, R., Hutchings, J. B., Beaulieu, M., et al. 2012, Proc. SPIE, 8442 , 84422R

Fabrycky, D., \& Tremaine, S. 2007, ApJ, 669, 1298
Fischer, D. A., Marcy, G. W., \& Spronck, J. F. P. 2014, ApJS, 210, 5 Ford, E. B., Kozinsky, B., \& Rasio, F. A. 2000, ApJ, 535, 385

Foreman-Mackey, D., Hogg, D. W., Lang, D., \& Goodman, J. 2013, PASP, 125,306

Fulton, B. J. 2017, PhD thesis, Univ. Hawai'i at Manoa

Fulton, B. J., Howard, A. W., Weiss, L. M., et al. 2016, ApJ, 830, 46

Fulton, B. J., \& Petigura, E. A. 2018, AJ, 156, 264

Fulton, B. J., Petigura, E. A., Blunt, S., \& Sinukoff, E. 2018, PASP, 130, 044504

Gaia Collaboration, Brown, A. G. A., Vallenari, A., et al. 2018, A\&A, 616, A1

Gray, R. O., Corbally, C. J., Garrison, R. F., McFadden, M. T., \& Robinson, P. E. 2003, AJ, 126, 2048

Hinkel, N. R., Mamajek, E. E., Turnbull, M. C., et al. 2017, ApJ, 848, 34

Høg, E., Fabricius, C., Makarov, V. V., et al. 2000, A\&A, 355, L27

Houdebine, E. R., Mullan, D. J., Paletou, F., \& Gebran, M. 2016, ApJ, 822, 97

Howard, A. W., Johnson, J. A., Marcy, G. W., et al. 2009, ApJ, 696, 75

Howard, A. W., Marcy, G. W., Johnson, J. A., et al. 2010, Sci, 330, 653

Huang, C. X., Petrovich, C., \& Deibert, E. 2017, AJ, 153, 210

Huber, D., Zinn, J., Bojsen-Hansen, M., et al. 2017, ApJ, 844, 102

Hunter, J. D. 2007, CSE, 9, 90

Isaacson, H., \& Fischer, D. 2010, ApJ, 725, 875

Johnson, J. A., \& Apps, K. 2009, ApJ, 699, 933

Johnson, J. A., Petigura, E. A., Fulton, B. J., et al. 2017, AJ, 154, 108

Kiseleva, L. G., Eggleton, P. P., \& Mikkola, S. 1998, MNRAS, 300, 292

Kopparapu, R. K., Ramirez, R., Kasting, J. F., et al. 2013, ApJ, 765, 131

Kosiarek, M. R., Crossfield, I. J. M., Hardegree-Ullman, K. K., et al. 2019, AJ, 157, 97

Kovács, G., Bakos, G., \& Noyes, R. W. 2005, MNRAS, 356, 557

Kurucz, R. L. 1970, SAOSR, 309, 93

Kurucz, R. L. 1993, SYNTHE Spectrum Synthesis Programs and Line Data (Cambridge, MA: Smithsonian Astrophysical Observatory)

López-Morales, M., Haywood, R. D., Coughlin, J. L., et al. 2016, AJ, 152, 204

Lovis, C., Dumusque, X., Santos, N. C., et al. 2011, arXiv:1107.5325

Luck, R. E. 2017, AJ, 153, 21

Maíz Apellániz, J., \& Weiler, M. 2018, A\&A, 619, A180

Mallama, A., Krobusek, B., \& Pavlov, H. 2017, Icar, 282, 19

Mann, A. W., Dupuy, T., Kraus, A. L., et al. 2019, ApJ, 871, 63

Marcy, G. W., \& Butler, R. P. 1992, PASP, 104, 270

Mason, B. D., Wycoff, G. L., Hartkopf, W. I., Douglass, G. G., \& Worley, C. E. 2001, AJ, 122, 3466

Mawet, D., Bond, C. Z., Delorme, J. R., et al. 2018, Proc. SPIE, 10703, 1070306

McKinney, W. 2010, in Proc. 9th Python in Science Conf., ed. S. van der Walt \& J. Millman (Austin, TX: SciPy), 51

Neves, V., Bonfils, X., Santos, N. C., et al. 2012, A\&A, 538, A25

Neves, V., Bonfils, X., Santos, N. C., et al. 2013, A\&A, 551, A36

Pepper, J., Kane, S. R., Rodriguez, J. E., et al. 2019, AJ, 159, 243

Pepper, J., Pogge, R. W., DePoy, D. L., et al. 2007, PASP, 119, 923

Petigura, E. A., Howard, A. W., Marcy, G. W., et al. 2017, AJ, 154, 107

Press, W. H., \& Rybicki, G. B. 1989, ApJ, 338, 277

Radovan, M. V., Lanclos, K., Holden, B. P., et al. 2014, Proc. SPIE, 9145, 91452B

Rein, H., \& Liu, S. F. 2012, A\&A, 537, A128

Rein, H., \& Tamayo, D. 2015, MNRAS, 452, 376

Schwarz, G. 1978, Ann. Statist., 6, 461

Schweitzer, A., Passegger, V. M., Cifuentes, C., et al. 2019, A\&A, 625, A68

Siverd, R. J., Beatty, T. G., Pepper, J., et al. 2012, ApJ, 761, 123

Speagle, J. S. 2019, MNRAS, 493, 3132

Stephenson, C. B. 1986, AJ, 92, 139

Stevens, D. J., \& Gaudi, B. S. 2013, PASP, 125, 933

Tange, O. 2011, login:, 36, 42, https://www.usenix.org/system/files/login/ articles/105438-Tange.pdf

van der Walt, S., Colbert, S. C., \& Varoquaux, G. 2011, CSE, 13, 22 van Leeuwen, F. 2007, A\&A, 474, 653

Virtanen, P., Gommers, R., Oliphant, T. E., et al. 2020, NatMe, 17, 261

Vogt, S. S., Allen, S. L., Bigelow, B. C., et al. 1994, Proc. SPIE, 2198, 362

Vogt, S. S., Radovan, M., Kibrick, R., et al. 2014, PASP, 126, 359

Weiss, L. M., \& Marcy, G. W. 2014, ApJL, 783, L6

Yee, S. W., Petigura, E. A., Fulton, B. J., et al. 2018, AJ, 155, 255

Yee, S. W., Petigura, E. A., \& von Braun, K. 2017, ApJ, 836, 77

Zacharias, N., Finch, C. T., Girard, T. M., et al. 2012, yCat, 3135, 0

Zsom, A., Seager, S., de Wit, J., \& Stamenković, V. 2013, ApJ, 778, 109 